\title{
Tuberculose: Questões sobre Reinfecção Exógena e Reativação Endógena ${ }^{1}$
}

S.M. RAIMUNDO², Faculdade de Medicina da Universidade de São Paulo, FMUSP, Av. Dr. Arnaldo 455, 01246-903 São Paulo, SP, Brasil

H.M. YANG ${ }^{3}$, Departamento de Matemática Aplicada, Instituto de Matemática, Estatística e Computação Científica, UNICAMP, Cx.P. 6065, 13081-970 Campinas, SP, Brasil.

\begin{abstract}
Resumo. Neste trabalho, formula-se um modelo matemático para descrever a dinâmica de transmissão da Tuberculose $(T B)$ e avaliar os fatores de impacto na sua epidemia e no seu controle. Com a finalidade de abordar vários aspectos da epidemiologia da $T B$ da forma mais simples possível considera-se que, uma vez infectado, o indíviduo pode progredir para a doença rapidamente ("fast" TB) ou de forma mais lenta, pela reativação endógena ( "slow" $T B$ ), ou então, adquirir uma nova infecção, pela reinfecção exógena. Os efeitos da vacinação e do tratamento são também analisados como estratégias de controle. Nossos resultados sugerem que o aumento na taxa de incidência da $T B$ é devido à reativação endógena (por exemplo, pela infecção pelo $H I V$ ) e/ou à reinfecção exógena.
\end{abstract}

\section{Introdução}

Tuberculose $(T B)$ é uma doença infecciosa causada pela bactéria Mycobacterium tuberculosis (MTB). TB é uma doença com aspectos singulares, com uma epidemiologia diferente da maioria das doenças infecciosas, devido ao tempo extremamente variável entre a doença e a prima-infecção com o $M T B$. Características da resposta imune de cada indivíduo indicarão se, após a prima-infecção, o indivíduo permanece no estado latente ou progride para a doença. Aproximadamente $90 \%$ dos indivíduos infectados permanecem no estado latente; $5-10 \%$ progridem rapidamente para a doença ( $T B$ primária) entre $2-5$ anos após a prima-infecção e $5 \%$ daqueles que inicialmente suprimiram a prima-infecção podem reativá-la durante a vida. A perda ou redução de imunidade, devido ao vírus da imunodeficiência humana $(H I V)$, por exemplo, pode aumentar a probabilidade de reativação em até $10 \%$ [2]. Os fatores relacionados à progressão para a doença ainda não estão bem definidos [1], ou seja, se é devida à reativação da prima-infecção (reativação endógena) ou a uma nova

\footnotetext{
${ }^{1}$ Apoio financeiro CNPq (Edital Universal 01/2002)

${ }^{2}$ silviamr@dim.fm.usp.br

${ }^{3}$ hyunyang@ime.unicamp.br
} 
cepa do $M T B$ (reinfecção exógena). A vacina $B C G$ (bacille Calmette-Guérin) é efetiva em reduzir a incidência da doença entre as crianças, mas não previne a infecção pelo $M T B$, nem mesmo protege os indivíduos adultos já vacinados. Assim, os indivíduos vacinados pela $B C G$ podem desenvolver $T B$ doença, tanto pela reinfecção exógena como pela reativação endógena.

Neste trabalho desenvolve-se um estudo teórico sobre a dinâmica da transmissão da Tuberculose $(T B)$ com o propósito de discutir o impacto da reinfecção exógena e reativação endógena na epidemia e no controle da $T B$, a eficiência da vacina $B C G$, supondo-se que todos os casos de $T B$ ativa recebem tratamento [4]. Propõe-se um modelo matemático para avaliar o impacto desses fatores na epidemia da $T B$ e no seu controle. Na Seção 2, desenvolve-se o modelo matemático com a dinâmica descrita por um sistema de equações diferenciais ordinárias não lineares. Na Seção 3 , apresenta-se um estudo do ponto de equilíbrio trivial e dos equilíbrios não triviais em duas situações endêmicas distintas. Por fim, na Seção 4, discute-se as implicações biológicas e apresenta-se as conclusões.

\section{Formulação do Modelo}

A população total, $N=X+X_{B C G}+L+X_{1}+T B+T B_{S}+T B_{R}$, é dividida em sete compartimentos, em cada instante de tempo $t$ : $X$, população de indivíduos suscetíveis a infecção pelo $M T B ; X_{B C G}$, indivíduos vacinados; $L$, indivíduos infectados pelo $M T B$ (prima-infecção), mas que não apresentam sintomas clínicos e são, portanto, não-infecciosos; $X_{1}$, indivíduos no estado latente; $T B$, indivíduos com $T B$ primária ("fast" ou "slow" $T B$ ); $T B_{S}$, indivíduos com $T B$ secundária (reinfecção exógena) e $T B_{R}$, indivíduos que recebem tratamento. Todos os parâmetros são positivos, e existe um fluxo de entrada constante ( $\Pi$ ) nas classes dos suscetíveis e vacinados $\left(X\right.$ e $\left.X_{B C G}\right)$. A dinâmica vital inclui a taxa de mortalidade natural, $\mu$, e a taxa de morte pela doença, $\alpha$. Os indivíduos suscetíveis tornam-se infectados pelo $M T B$ através do contacto com os indivíduos com TB doença. Uma vez infectados, esses indivíduos podem desenvolver $T B$ por (a) progressão direta "fast" TB; (b) reativação endógena - "slow" TB; (c) adquirir uma nova infecção de outros indivíduos, caracterizando a reinfecção exógena ou então, permanecer no estado estado latente, $X_{1}$, sem nunca desenvolver a doença. Por sua vez, os indivíduos vacinados podem também infectar-se pelo $M T B$, mas com uma intensidade menor, $q \beta$, com $0 \leq q \leq 1$.

O período médio para desenvolver "fast" $T B$ é $\omega_{1}^{-1}$ e para a "slow" $T B$ é $\left(\omega_{2}+\lambda\right)^{-1}$. Por suposição, o tratamento é cumprido integralmente por todos os pacientes $\left(T B\right.$ e $\left.T B_{S}\right)$. Define-se: $(1-p)$, a proporção de indivíduos recrutados

para a classe dos suscetíveis $X ; p$, a proporção de indivíduos que entram na classe dos vacinados $\left(X_{B C G}\right) ; \beta$, o coeficiente de transmissão do $M T B ; r$, a taxa na qual os indivíduos suprimem a prima-infecção ou taxa de perda de imunidade pela vacina, e retornam para a classe dos vacinados. ; $\omega_{1}$, a taxa na qual os indivíduos infectados desenvolvem "fast" $T B ; \omega_{2}$, a taxa na qual os indivíduos infectados progridem para a classe dos indivíduos latentes $\left(X_{1}\right) ; \lambda$, a taxa na qual os indivíduos infectados desenvolvem $T B$ pela reativação endógena ("slow" $T B$ ); $\beta$ ', a taxa na 
qual os indivíduos infectados desenvolvem $T B$ pela reinfecção exógena; $\xi$, a taxa de tratamento e $\sigma$, a taxa na qual os indivíduos tratados retornam para a classe $X_{1}$. O modelo é descrito pelo seguinte sistema de equações diferencias ordinárias

$$
\begin{cases}\frac{d X}{d t} & =(1-p) \Pi-\beta X\left(T B+T B_{S}\right)-\mu X \\ \frac{d X_{B C G}}{d t} & =p \Pi-q \beta X_{B C G}\left(T B+T B_{S}\right)-\mu X_{B C G}+r L \\ \frac{d L}{d t} & =\beta X\left(T B+T B_{S}\right)+q \beta X_{B C G}\left(T B+T B_{S}\right)-\left(\omega_{1}+\omega_{2}+r+\mu\right) L \\ \frac{d X_{1}}{d t} & =-\lambda X_{1}-\beta^{\prime} X_{1}\left(T B+T B_{S}\right)+\sigma T B_{R}+w_{2} L-\mu X_{1} \\ \frac{d T B}{d t} & =\lambda X_{1}+w_{1} L-(\xi+\mu+\alpha) T B \\ \frac{d T B_{S}}{d t} & =\beta^{\prime} X_{1}\left(T B+T B_{S}\right)-(\xi+\mu+\alpha) T B_{S} \\ \frac{d T B_{R}}{d t} & =\xi\left(T B+T B_{S}\right)-(\mu+\sigma) T B_{R},\end{cases}
$$

onde $\frac{d N}{d t}=\Pi-\mu N-\alpha\left(T B+T B_{S}\right)$. Para $N>\frac{\Pi}{\mu}$, a população total é decrescente, isto é, $\frac{d N}{d t}<0$. Se $\alpha=0$, então $\frac{d N}{d t}=\Pi-\mu N$, e tem-se a assíntota $N(t) \rightarrow \frac{\Pi}{\mu}$, quando $t \rightarrow \infty$. Se $\alpha=0$ e $\Pi=\mu N$, então $\frac{d N}{d t}=0$, e a população total é constante, $N(0)$.

\section{Estabilidade do Equilíbrio Trivial e o Número de Reprodutibilidade, $\mathcal{R}_{p}$}

O número de reprodutibilidade basal, $\mathcal{R}_{0}$, é um parâmetro epidemiológico muito útil para quantificar a transmissão de um patógeno, sendo definido como o número médio de infecções secundárias produzidas por um indivíduo infectado quando introduzido numa população inteiramente suscetível, na ausência de qualquer tipo de heterogeneidade. Sabe-se que $\mathcal{R}_{0}$ é um valor limiar e sempre que $\mathcal{R}_{0} \leq 1$ o equilíbrio livre da doença é globalmente assintoticamente estável (G.A.E.), e a epidemia não ocorre. Para $\mathcal{R}_{0}>1$, existe um único equilíbrio endêmico localmente assintoticamente estável (L.A.E.). Assim, a epidemia persiste na população independentemente das condições iniciais do sistema e a expressão analítica para $\mathcal{R}_{0}$ pode ser calculada pelos resultados da análise do equilíbrio trivial. Entretanto, numa população onde existem a vacinação e a reinfecção, o limiar dado por $\mathcal{R}_{0}$ é substituído por um outro limiar, aqui definido como valor de reprodutibilidade, $\mathcal{R}_{p}$.

$\mathrm{O}$ sistema (2.1) tem sempre o equilíbrio trivial $P_{0}^{*}=\left(X^{*}, X_{B C G}^{*}, 0,0,0,0,0\right)$, ou livre da doença, com as coordenadas $X^{*}=(1-p) \Pi / \mu$ e $X_{B C G}^{*}=p \Pi / \mu$. A estabilidade do equilíbrio trivial é verificada através da matriz jacobiana do sistema (2.1) calculada em $P_{0}^{*}$. A matriz jacobiana tem sete autovalores: $\lambda_{1}=\lambda_{2}=-\mu \mathrm{e}$ $\lambda_{3}=-(\mu+\alpha+\xi)$ com os outros quatro dados pelas raízes do seguinte polinômio característico,

$$
P(\lambda)=\lambda^{4}+a_{1} \lambda^{3}+a_{2} \lambda^{2}+a_{3} \lambda+a_{4}
$$


com

$$
\left\{\begin{aligned}
a_{1}= & \left(\omega_{1}+\omega_{2}+2 \mu+r+\lambda\right)+(\mu+\alpha+\xi)+(\mu+\sigma) \\
a_{2}= & \mu(\mu+\sigma)\left(\omega_{1}+\omega_{2}+2 \mu+r+\lambda\right)+\mu(\mu+\alpha+\xi)(\mu+\sigma)+ \\
& \mu\left(\omega_{1}+\omega_{2}+2 \mu+r+\lambda\right)(\mu+\alpha+\xi)+\mu\left[\left(\omega_{1}+\omega_{2}+\mu+r\right)(\lambda+\mu)\right]- \\
& \omega_{1} \Pi \beta[1-p(1-q)] \\
a_{3}= & \mu\left(\omega_{1}+\omega_{2}+\mu+r\right)\{(\lambda+\mu)(2 \mu+\sigma+\alpha+\xi)+(\mu+\sigma)(\mu+\alpha+\xi)\}+ \\
& \mu^{2}(\lambda+\mu+\sigma)(\mu+\alpha+\xi)+\mu \sigma \lambda(\mu+\alpha)- \\
& \Pi \beta[1-p(1-q)]\left[\lambda\left(\omega_{1}+\omega_{2}\right)+\mu \omega_{1}+(\mu+\sigma) \omega_{1}\right] \\
a_{4}= & \mu\left(\omega_{1}+\omega_{2}+\mu+r\right)\{\lambda[(\mu+\sigma)(\mu+\alpha)+\mu \xi]+\mu(\mu+\sigma)(\mu+\alpha+\xi)\}- \\
& \Pi \beta[1-p(1-q)](\mu+\sigma)\left[\lambda\left(\omega_{1}+\omega_{2}\right)+\mu \omega_{1}\right] .
\end{aligned}\right.
$$

Para avaliar a estabilidade de $P_{0}^{*}=\left(X^{*}, X_{B C G}^{*}, 0,0,0,0,0\right)$, o critério de RouthHurwitz determina que se $a_{1}>0, a_{3}>0, a_{4}>0$ e $a_{1} a_{2} a_{3}>a_{3}^{2}+a_{1}^{2} a_{4}$, então todos os autovalores associados ao polinômio (3.1) são negativos (se reais) ou têm parte real negativa (se complexos). Verificando tais condições, conclui-se que o critério de Routh-Hurwitz é satisfeito quando $a_{4}>0$. Ou seja, $P_{0}^{*}$ é G.A.E. sempre que $\beta<\beta_{p}$, com a taxa de contato limiar $\beta_{p}$ dada por

$\beta_{p}=\frac{\left(\omega_{1}+\omega_{2}+\mu+r\right) \mu\{\lambda[(\mu+\sigma)(\mu+\alpha)+\mu \xi]+\mu(\mu+\sigma)(\mu+\alpha+\xi)\}}{(\mu+\sigma) \Pi[1-p(1-q)]\left[\lambda\left(\omega_{1}+\omega_{2}\right)+\mu \omega_{1}\right]}$.

Caso contrário, $P_{0}^{*}$ é instável. Desta forma, o número de reprodutibilidade, $\mathcal{R}_{p}$, é então definido por $\mathcal{R}_{p}=\beta / \beta_{p}$. Assim, quando $\mathcal{R}_{p}<1$, o equilíbrio trivial $P_{0}^{*}$ é G.A.E. e a doença é eliminada na população. Quando $\mathcal{R}_{p}>1$, o equilíbrio trivial $P_{0}^{*}$ é instável e a epidemia deflagra. Observe que $\mathcal{R}_{p}$ não depende do parâmetro $\beta^{\prime}$, porém a reinfecção exógena aumenta o esforço que deve ser feito para a infecção ser eliminada da população, pois aumenta a prevalência da doença. Quando $p=0$, tem-se o número de reprodutibilidade basal, $\mathcal{R}_{0}=\beta / \beta_{0}$.

\section{Equilíbrio Endêmico para $\beta^{\prime}=0$ e $\beta^{\prime}=\beta$}

Reescrevendo o sistema (2.1), da seguinte forma:

$$
\begin{cases}\frac{d X}{d t} & =(1-p) \Pi-\beta X T B_{T O T}-\mu X \\ \frac{d X_{B C G}}{d t} & =p \Pi-q \beta X_{B C G} T B_{T O T}-\mu X_{B C G}+r L \\ \frac{d\left(X+X_{B C G}+L\right)}{d t} & =\Pi-\mu\left(X+X_{B C G}+L\right)-\left(\omega_{1}+\omega_{2}\right) L \\ \frac{d\left(X_{1}+T B+T B_{S}\right)}{d t} & =\sigma T B_{R}+\left(\omega_{1}+\omega_{2}\right) L-(\xi+\mu+\alpha) T B_{T O T}-\mu X_{1} \\ \frac{d T B_{T O T}}{d t} & =\lambda X_{1}+\beta^{\prime} X_{1} T B_{T O T}+w_{1} L-(\xi+\mu+\alpha) T B \\ \frac{d T B_{R}}{d t} & =\xi T B_{T O T}-(\mu+\sigma) T B_{R},\end{cases}
$$

com $T B_{T O T}^{*}=T B+T B_{S}$, determina-se o ponto de equilíbrio não trivial, ou equilíbrio endêmico, $P^{*}=\left(X^{*}, X_{B C G}^{*}, L^{*}, X_{1}^{*}, T B_{T O T}^{*}, T B_{R}^{*}\right)$, com as coordenadas dadas por 


$$
\left\{\begin{aligned}
X^{*} & =\frac{(1-p) \Pi}{\beta T B_{T O T}^{*}+\mu} \\
X_{B C G}^{*} & =\frac{p \Pi+r L^{*}}{q \beta T B_{T O T}^{*}+\mu} \\
L^{*} & =\frac{\left[\Pi \beta^{2} q T B_{T O T}^{*}+\Pi \beta \mu+\mu p \Pi \beta(q-1)\right] T B_{T O T}^{*}}{\left[\left(q \beta T B_{T O T}^{*}+\mu\right)\left(\omega_{1}+\omega_{2}+\mu\right)+\mu r\right]\left(\beta T B_{T O T}^{*}+\mu\right)} \\
T B_{R}^{*} & =\frac{\xi T B_{T O T}^{*}}{(\mu+\sigma)} \\
X_{1}^{*} & =\frac{\sigma T B_{R}^{*}+\left(\omega_{1}+\omega_{2}\right) L^{*}-(\xi+\mu+\alpha) T B_{T O T}^{*}}{\mu} \\
X_{1}^{*} & =\frac{(\xi+\mu+\alpha) T B_{T O T}^{*}-\mu \omega_{1} L^{*}}{\lambda+\beta^{\prime} T B_{T O T}^{*}},
\end{aligned}\right.
$$

onde o valor de $T B_{T O T}^{*}$ é determinado pela equação:

$$
\begin{array}{r}
\left(\lambda+\beta^{\prime} T B_{T O T}^{*}\right)\left[\sigma T B_{R}^{*}+\left(\omega_{1}+\omega_{2}\right) L^{*}-(\xi+\mu+\alpha) T B_{T O T}^{*}\right] \\
-\mu(\xi+\mu+\alpha) T B_{T O T}^{*}-\mu \omega_{1} L^{*}=0
\end{array}
$$

Substituindo-se (4.2) na equação (4.3) obtém-se $T B_{T O T}^{*}=0$ ou a solução nãonula, $T B_{\text {TOT }}^{*} \neq 0$, através do polinômio:

$$
A_{0}^{*}\left(\beta^{\prime}\right) \quad\left(T B_{T O T}^{*}\right)^{3}+A_{1}^{*}\left(\beta^{\prime}\right) \quad\left(T B_{T O T}^{*}\right)^{2}+A_{2}^{*}\left(\beta^{\prime}\right) T B_{T O T}^{*}+A_{3}^{*}\left(\beta^{\prime}\right)=0,
$$

com os coeficientes em função de $\beta^{\prime}$ dadas por

$$
\left\{\begin{aligned}
A_{0}^{*}\left(\beta^{\prime}\right) & =\beta^{\prime}\left[\frac{(\mu+\alpha)(\mu+\sigma)+\mu \xi}{(\mu+\sigma)}\right] q \beta^{2}\left(\omega_{1}+\omega_{2}+\mu\right) \\
A_{1}^{*}\left(\beta^{\prime}\right) & =\beta^{\prime}\left[\frac{(\mu+\alpha)(\mu+\sigma)+\mu \xi}{(\mu+\sigma)}\right]\left[\beta \mu\left(\omega_{1}+\omega_{2}+\mu\right)(1+q)+\beta \mu r\right] \\
& +\left[\frac{\lambda[(\mu+\alpha)(\mu+\sigma)+\mu \xi]+\mu(\mu+\sigma)(\mu+\sigma+\xi)}{(\mu+\sigma)}\right] q \beta^{2}\left(\omega_{1}+\omega_{2}+\mu\right)-\beta^{\prime}\left(\omega_{1}+\omega_{2}\right) \Pi q \beta^{2} \\
A_{2}^{*}\left(\beta^{\prime}\right) & =\beta^{\prime}\left[\frac{(\mu+\alpha)(\mu+\sigma)+\mu \xi}{(\mu+\sigma)}\right] \mu^{2}\left(\omega_{1}+\omega_{2}+\mu+r\right) \\
& +\left[\frac{\lambda[(\mu+\alpha)(\mu+\sigma)+\mu \xi]+\mu(\mu+\sigma)(\mu+\sigma+\xi)}{(\mu+\sigma)}\right]\left[\beta \mu\left(\omega_{1}+\omega_{2}+\mu\right)(1+q)+\beta \mu r\right] \\
& -\beta^{\prime}\left(\omega_{1}+\omega_{2}\right) \Pi \beta \mu[1-p(1-q)]-\left[\lambda\left(\omega_{1}+\omega_{2}\right)+\mu \omega_{1}\right] \Pi \beta^{2} q \\
A_{3}^{*}\left(\beta^{\prime}\right) & =\left[\frac{\lambda[(\mu+\alpha)(\mu+\sigma)+\mu \xi]+\mu(\mu+\sigma)(\mu+\sigma+\xi)}{(\mu+\sigma)}\right] \mu\left(\omega_{1}+\omega_{2}+\mu+r\right) \\
& -\left[\lambda\left(\omega_{1}+\omega_{2}\right)+\mu \omega_{1}\right] \Pi \beta[1-p(1-q)] .
\end{aligned}\right.
$$

Quando $T B_{T O T}^{*}=0$, tem-se o equilíbrio trivial, cuja análise de estabilidade já foi descrita anteriormente.

Na situação endêmica, o equilíbrio não trivial é obtido determinando os valores de $T B_{T O T}^{*}$ como raíz do polinômio (4.4). Assim, é possível determinar as condições nas quais o equilíbrio endêmico, $P^{*}=\left(X^{*}, X_{B C G}^{*}, L^{*}, X_{1}^{*}, T B_{T O T}^{*}, T B_{R}^{*}\right)$, é único e biologicamente viável (todas as coordenadas são positivas). Alem disso, como cada sub-população dada por (4.2) está definida como função de $T B_{T O T}^{*}$, para mostrar que existe o equilíbrio não trivial positivo para o sistema (2.1), é suficiente mostrar que $T B_{T O T}^{*}$ é positiva para o polinômio (4.4). Esta análise será apresentada para duas situações epidêmicas. Primeiro, a infecção é mantida somente pela infecção primária, isto é, $\beta^{\prime}=0$. Segundo, a reinfecção exógena e a infecção primária ocorrem a uma mesma taxa, ou seja, $\beta^{\prime}=\beta$. A reativação endógena ocorre em ambos os casos. No último caso, fazendo $q=0$ e $q=1$, investiga-se uma resposta imune 
induzida pela vacina nos casos extremos. Usando o Teorema do Valor Intermediário, e as simulações numéricas, generalizamos as condições nas quais $T B_{T O T}^{*}$ é positiva e onde o ponto de equilíbrio não trivial é estável.

Observe que o sistema (2.1) tem sempre o equilíbrio livre da doença, enquanto o equilíbrio endêmico depende sempre dos valores dos coeficientes de transmissão $\left(\beta^{\prime} \mathrm{e}\right.$ $\beta$ ) e do valor da taxa de reativação endógena $(\lambda)$. Mostramos, a seguir, que quando os valores de $\beta$ e $\lambda$ variam, múltiplos equilíbrios endêmicos podem ocorrer para o sistema (2.1). De acordo com esta variação investiga-se a existência dos possíveis equilíbrios endêmicos, considerando-se os dois casos mencionados anteriormente: $\beta^{\prime}=0$ e $\beta^{\prime}=\beta$. A estabilidade destes pontos é obtida através de procedimentos numéricos.

A partir de agora as análises serão desenvolvidas substituindo-se os valores de $\beta^{\prime}=0$, ou de $\beta^{\prime}=\beta$, nos coeficientes (4.5) do polinômio (4.4).

\subsection{A não ocorrência da reinfecção exógena $\left(\beta^{\prime}=0\right)$}

Fazendo $\beta^{\prime}=0$ em (4.5), o polinômio (4.4) é dado por

$$
A_{1}\left(T B_{T O T}^{*}\right)^{2}+A_{2}\left(T B_{T O T}^{*}\right)+A_{3}=0 .
$$

De (4.5) tem-se que $A_{1}=A_{1}^{*}(0)>0$. Além disso, de (3.2) observa-se que $A_{3}=$ $A_{3}^{*}(0)=a_{4}$. Portanto, existe uma única raiz real positiva, $T B_{T O T}^{*}$, para o polinômio (4.6), independente do sinal de $A_{2}=A_{2}^{*}(0)$. Assim, existe um único ponto de equilíbrio endêmico, $P^{*}=\left(X^{*}, X_{B C G}^{*}, L^{*}, X_{1}^{*}, T B_{T O T}^{*}, T B_{R}^{*}\right)$, biologicamente viável, que é L.A.E. para $\mathcal{R}_{p}>1$. Ou seja, a doença pode ser mantida na população pela reativação endógena, mesmo que o tratamento seja cumprido integralmente pelos pacientes. Isto sugere que, por ser uma doença oportunista, a infecção pelo $H I V$ é um fator importante na reemergência da $T B$.

\subsection{A ocorrência da reinfecção exógena $\left(\beta^{\prime}=\beta\right)$}

Neste caso considera-se que a reinfecção exógena e a infecção ocorrem a uma mesma taxa, quando a eficiência da vacina é $100 \%(q=0)$ ou nula $(q=1)$.

\subsubsection{Vacina efetiva $(q=0)$}

Fazendo-se $\beta^{\prime}=\beta$ e $q=0$ nos coeficientes (4.5) do polinômio (4.4), obtém-se

$$
A_{4}\left(T B_{T O T}^{*}\right)^{2}+A_{5}\left(T B_{T O T}^{*}\right)+A_{6}=0,
$$

com $A_{4}=A_{1}^{*}(\beta)>0$. Primeiro, os valores de $\beta$ que anulam os coeficientes $A_{5}=$ $A_{2}^{*}(\beta)$ e $A_{6}=A_{3}^{*}(\beta)$ são determinados e designados por $\beta_{A_{5}}$ e $\beta_{A_{6}}$, respectivamente. Conseqüentemente, o valor de $\beta$ para o qual $T B_{T O T}^{*}$ é positiva pode ser, então, determinado observando-se a posição relativa entre $\beta_{A_{5}}$ e $\beta_{A_{6}}$, dada por

$$
\beta_{A_{5}}-\beta_{A_{6}}=a_{0}^{\prime} \lambda^{2}+a_{1}^{\prime} \lambda+a_{2}^{\prime},
$$


com os coeficientes $a_{0}^{\prime}>0, a_{1}^{\prime}>0$ e $a_{2}^{\prime}<0$ (por serem extensas, as expressões desses coeficientes são omitidas). $\mathrm{O}$ polinômio (4.8) tem exatamente uma única solução positiva, designada por $\lambda_{A_{56}}^{+}$. De acordo com as variações de $\lambda$ e de $\beta$, os seguintes casos podem ocorrer:

Caso A) Para $\lambda>\lambda_{A_{5,6}}^{+}$tem-se $\beta_{A_{5}}-\beta_{A_{6}}>0$.

Se $\beta<\beta_{A_{6}}$, isto é, quando $\mathcal{R}_{A_{6}}^{*}=\beta / \beta_{A_{6}}<1$, o sistema (2.1) tem somente o equilíbrio livre da doença, G.A.E. Se $\mathcal{R}_{A_{6}}^{*}>1$, existe uma única solução positiva $T B_{T O T}^{*}$ para (4.7) e, portanto, um único equilíbrio endêmico, L.A.E., para o sistema (2.1). Este fato sugere que, mesmo com a eficácia vacinal de $100 \%$, a reinfecção exógena pode ter um papel importante na incidência da $T B$, desde que o esforço ( $\beta$ ) para manter a infecção na população cresça. Observe que $\mathcal{R}_{\bar{A}_{3}}^{*}>\mathcal{R}_{p}$, onde $\mathcal{R}_{p}=\frac{\beta}{\beta_{p}}$, com o limiar $\beta_{p}$ definido por (3.3).

Caso B) Para $0<\lambda<\lambda_{A_{5,6}}^{+}$tem-se $\beta_{A_{5}}-\beta_{A_{6}}<0$.

Se $\beta<\beta_{A_{5}}$, o sistema (2.1) tem somente o equilíbrio livre da doença G.A.E. Se $\beta_{A_{5}}<\beta<\beta_{A_{6}}$, então existem duas soluções positivas $T B_{T O T}^{*}$ para (4.7) e, portanto, dois equilíbrios endêmicos positivos, para o sistema (2.1), o maior deles L.A.E. Se $\mathcal{R}_{A_{6}}^{*}>1$, então existe uma única solução positiva $T B_{T O T}^{*}$ para (4.7) e, portanto, um único equilíbrio endêmico positivo, L.A.E., para o sistema (2.1). Esta análise é apresentada com detalhes em [5].

\subsubsection{Eficácia nula da vacina $(q=1)$}

Fazendo $\beta^{\prime}=\beta$ e $q=1$, nos coeficientes (4.5) do polinômio (4.4), obtém-se

$$
A_{7}\left(T B_{T O T}^{*}\right)^{3}+A_{8}\left(T B_{T O T}^{*}\right)^{2}+A_{9}\left(T B_{T O T}^{*}\right)+A_{10}=0 .
$$

Desde que $A_{7}=A_{0}^{*}(\beta)>0$, devemos então analisar a posição relativa dos coeficientes $A_{8}=A_{1}^{*}(\beta), A_{9}=A_{2}^{*}(\beta)$ e $A_{10}=A_{3}^{*}(\beta)$ em três casos distintos.

Primeiro, determina-se os valores de $\beta$ que anulam os coeficientes $A_{9}$ e $A_{10}$, agora designados, respectivamente, por $\beta_{A_{9}}$ e $\beta_{A_{10}}$. De forma análoga ao que foi feito anteriormente, calcula-se a posição relativa desses valores, fazendo

$$
\beta_{A_{9}}-\beta_{A_{10}}=b_{0}^{\prime} \lambda^{2}+b_{1}^{\prime} \lambda+b_{2}^{\prime},
$$

com $b_{0}^{\prime}>0, b_{1}^{\prime}>0$ e $b_{2}^{\prime}<0$ (as expressões dos coeficientes são omitidas por serem extensas); donde conclui-se que o polinômio (4.10) tem exatamente uma única solução positiva, neste caso designada por $\lambda_{9,10}^{+}$. Disto segue que,

i) Para $\lambda>\lambda_{A_{9,10}}^{+}$tem-se $\beta_{A_{9}}-\beta_{A_{10}}>0$

ii) Para $0<\lambda<\lambda_{A_{9,10}}^{+}$tem-se $\beta_{A_{9}}-\beta_{A_{10}}<0$. 
Segundo, investiga-se a posição relativa entre $\beta_{A_{8}}$ e $\beta_{A_{10}}$, valores que anulam os coeficientes $A_{8}$ e $A_{10}$, fazendo

$$
\beta_{A_{8}}-\beta_{A_{10}}=c_{0}^{\prime} \lambda^{2}+c_{1}^{\prime} \lambda+c_{2}^{\prime},
$$

donde $c_{0}^{\prime}>0, c_{1}^{\prime}>0$ e $c_{2}^{\prime}=\left(\omega_{1}+\omega_{2}+\mu\right)\left[\frac{(\mu+\sigma)(\mu+\alpha)+\mu \xi}{(\mu+\sigma)}\right] \mu^{2} \omega_{1}+b_{2}^{\prime}$.

Por fim, dos coeficientes $A_{8}$ e $A_{9}$ tem-se,

$$
\beta_{A_{8}}-\beta_{A_{9}}=d_{0}^{\prime} \lambda^{2}+d_{1}^{\prime} \lambda+d_{2}^{\prime},
$$

$\operatorname{com} d_{0}^{\prime}>0, d_{1}^{\prime}>0$ e $d_{2}^{\prime}=\left(\omega_{1}+\omega_{2}+\mu\right)\left[\frac{(\mu+\sigma)(\mu+\alpha)+\mu \xi}{(\mu+\sigma)}\right] \mu^{2}\left(2 \omega_{1}+\omega_{2}\right)+b_{2}^{\prime}$.

Importante observar que as expressões dos coeficientes são omitidas por serem extensas e que, como $b_{2}^{\prime}<0$, o sinal de $c_{2}^{\prime}$ e de $d_{2}^{\prime}$ nos polinômios (4.11) e (4.12) deverá ser investigado.

Assim, as raízes de (4.11) e (4.12) podem ser determinadas de acordo com a seguinte variação do sinal dos coeficientes $c_{2}^{\prime}$ e $d_{2}^{\prime}$ :

(i) se $c_{2}^{\prime}>0$ e $d_{2}^{\prime}>0$ então as soluções para os dois polinômios são negativas. Este caso, além de não ter sentido epidemiológico, não satisfaz a hipótese de todos os parâmetros serem positivos, no caso, $\lambda<0$.

(ii) se $d_{2}^{\prime}<0$ e $c_{2}^{\prime}<0$ então cada polinômio tem uma solução negativa e outra positiva.

Tomando $\lambda_{8,10}^{+}$como a solução positiva de (4.11) e $\lambda_{8,9}^{+}$a solução positiva de (4.12), verifica-se que $\lambda_{8,10}^{+}>\lambda_{8,9}^{+}$. Por fim, juntando todas as possíveis combinações das posições relativas de $\lambda$, com as posições relativas de $\beta$, através de (4.10), determina-se a existência de todos possíveis equilíbrios endêmicos. Assim, o sistema (2.1) pode apresentar os seguintes pontos de equilíbrio: (a) o equilíbrio livre da doença G.A.E.; (b) um único equilíbrio endêmico L.A.E.; (c) dois equilíbrios endêmicos, com o menor deles L.A.E. (vide detalhes em [5]) ou (d) até três equilíbrios endêmicos, com um deles L.A.E. e os outros dois complexos ou reais negativos.

Interessante notar que a existência do equilíbrio livre da doença depende da posição relativa entre $\beta$ e $\lambda$, enquanto a situação de existência de um único equilíbrio endêmico é definida apenas pelo valor do limiar $\mathcal{R}_{A_{10}}^{*}$. Assim, se $\beta>\beta_{A_{10}}$, ou seja, se $\mathcal{R}_{A_{10}}^{*}=\beta / \beta_{A_{10}}>1$, então existe um único equilíbrio endêmico L.A.E. para o sistema $(2.1)$.

É importante ressaltar que a análise feita para o sistema (2.1), em ambos os casos $q=0$ e $q=1$, mostra a existência de nenhuma, uma, duas ou até três soluções positivas para $T B_{T O T}^{*}$. Se cada condição é satisfeita, é possível existirem até três soluções positivas $T B_{T O T}^{*}$ para o polinômio (4.4). Assim, a análise numérica mostra quando o equilíbrio endêmico $P^{*}=\left(X^{*}, X_{B C G}^{*}, L^{*}, X_{1}^{*}, T B_{T O T}^{*}, T r^{*}\right)$ dado pela equação (4.2) é estável ou não.

\section{Implicações Epidemiológicas e Conclusões}

O objetivo deste trabalho é o estudo da dinâmica da transmissão da $T B$ quando a vacina $B C G$ e o tratamento são adotados como estratégias de controle. Embora 
aproximadamente $95 \%$ dos pacientes com $T B$, que completam o tratamento, retornem para o estado latente, infelizmente as vacinas não são provavelmente $100 \%$ eficientes e não atuam como prevenção da doença. $B C G$ pode ser efetiva em reduzir a incidência da $T B$, mas é ineficiente em proteger os adultos contra a doença. Em nosso modelo, considera-se a hipótese de que todos os indivíduos que cumprem o tratamento retornam para a classe dos indivíduos latentes, $X_{1}$. Baseado nesta suposição, o modelo permite que os efeitos da vacina sejam investigados.

Analisando o número de reprodutibilidade, conclui-se que o esforço para manter $\mathcal{R}_{p}<1$ é menor quando a vacina é $100 \%$ efetiva, ou seja, quando $q=0$. Este fato sugere que a erradicção poderia ser possível se a proteção induzida pela vacina ocorresse em todos os indivíduos vacinados. Sob o ponto de vista da reativação endógena, tomando $\lambda=0$ e $\lambda=\infty$ na equação (3.3), tem-se para o número de reprodutibilidade $\mathcal{R}_{p}=\frac{\beta}{\beta_{p}(\lambda=0)}$ e $\mathcal{R}_{p}=\frac{\beta}{\beta_{p}(\lambda \rightarrow \infty)}$. Mostra-se facilmente que $\beta_{p}(\lambda=0)-\beta_{p}(\lambda \rightarrow \infty)<0$, ou seja, o valor limiar $\beta_{p}(\lambda)$ decresce com o taxa de reativação endógena $\lambda$. Portanto, o decréscimo de $\beta_{p}(\lambda)$ com $\lambda$ tem como efeito aumento do número de reprodutibilidade $\mathcal{R}_{p} \operatorname{com} \lambda$, o que implica na possibilidade de aumentar a incidência (ou ressurgência em regiões livres) da $T B$. Por isso, regiões (ou países) outrora livres de $T B$ estão sofrendo a re-emrgência desta infecção devido à endemia de síndrome da imunodeficiência humana $(A I D S)$.

Portanto, mesmo que as estratégias de controle, tais como vacinação e tratamento, reduzissem a transmissão pelo $M T B$, a incidência da $T B$ pode aumentar devido à reativação endógena. Isto corrobora o fato de que a redução na resposta imune dos indivíduos devido ao $H I V$ aumenta a probabilidade de reativação da $T B$ [3]. Além disso, se a reativação endógena cresce, então $\mathcal{R}_{p}$ cresce. Portanto, se a taxa de reativação endógena é alta, o esforço para a infecção ser mantida na população pela reinfecção exógena ou/e infecção pode ser menor. É necessário diminuir a taxa de reativação para diminuir $\mathcal{R}_{0}$. Isto mostra que a reativação endógena tem um papel importante na epidemia da $T B$.

Finalmente, estes resultados sugerem que, para erradicar a $T B$, seria necessário que todos os indivíduos fossem vacinados com vacinas $100 \%$ efetivas $(q=0, p=1)$. Assim, $\mathcal{R}_{p=1}=1$, independentemente da reativação endógena ou/e reinfecção exógena.

Vale ressaltar que o modelo matemático aqui apresentado apresenta múltiplos equilíbrios endêmicos devido à super-infecção ou reinfecção exógena. Assim, para a erradicação da $T B$ não basta baixar $\mathcal{R}_{p}$ a valores menores que um, mas sim considerar um outro valor limiar, $\mathcal{R}_{p}^{*}$. Portanto, se é possível baixar apenas $\mathcal{R}_{p}$, deve-se também baixar a prevalência da doença no domínio de atração do ponto de equilíbrio trivial, que é a alternativa para a erradicação da doença [5].

O propósito deste trabalho é muito específico. Para estudar a dinâmica da transmissão da $T B$, formulamos um modelo básico, tão simples quanto possível, considerando simultaneamente vários aspectos da epidemiologia da $T B$, tais como infecções endógena e exógena, "fast" e "slow" $T B$, vacina $B C G$ e tratamento. A desvantagem deste modelo é que não estamos considerando um sério problema de saúde pública, que é o abandono ao tratamento pelos pacientes. Contudo, esta formulação permitiu um estudo teórico e conclusões analíticas importantes. Assim, este modelo é a base de um estudo complexo, tais como os efeitos da resistência 
a drogas e o aparecimento da $T B$ resistente a multi-drogas $(M D R-T B)$. Neste sentido, o próximo propósito é o de incorporar no modelo as dificuldades em tratar pacientes com $T B$ doença e o não cumprimento do tratamento, e acreditamos que simulações numéricas possam corroborar nossas conclusões analíticas.

\begin{abstract}
This work is concerned with a theoretical framework for assessing the transmission dynamics of Tuberculosis $(T B)$. We propose a mathematical model to evaluate the impact of factors on $T B$ epidemic and its control, by including into the model both effects of vaccination and treatment of active TB cases. Once infected with Mycobacterium tuberculosis, an individual can be either "a slow" (endogenous infection) or "a fast" progressor to TB. Latent infected individuals may be reinfected acquiring a new infection from another infectious individuals (exogenous reinfection). Our results suggest that even if effective control strategies could have a significant effect on reducing $T B$ transmission, the endogenous infection, due to mainly $H I V$ infection, increases $T B$ incidence. Besides, we also observed that exogenous reinfection may also play an important role in the transmission of $T B$.
\end{abstract}

\title{
Referências
}

[1] B.M. Murphy, B.H. Singer e D. Kirschner, On treatment of tuberculosis in heterogenous populations, J. Theoretical Biology, 223, No. 4 (2003), 391-404 .

[2] S.M. Raimundo, H.M. Yang, R.C. Bassanezi e M.A.C. Ferreira, The Attraction Basins and the Assessment of the Transmission Coefficients for HIV and $M$. tuberculosis Infections among Women Inmates, J. Biol. Syst., 10, No. 1 (2002), 61-83.

[3] S.M. Raimundo, H.M. Yang, A.B. Engel e R.C. Bassanezi, An approach to estimating the Transmission Coefficients for AIDS and for Tuberculosis, Systems Analysis Modelling Simulation, 43, No. 4 (2003), 423-442.

[4] S.M. Raimundo e H.M. Yang, A mathematical model for exogenous reinfection and control of Tuberculosis transmission, Abstract Book: Computational and Mathematical Population Dynamics (CMPD), Trento, Italy, pg. 129, 2004.

[5] S.M. Raimundo e H.M. Yang, Dinâmica de transmissão das moléstias infecciosas: múltiplas infecções e período de latência, TEMA Tend. Mat. Apl. Comput., 6, No. 1 (2005), 131-140. 